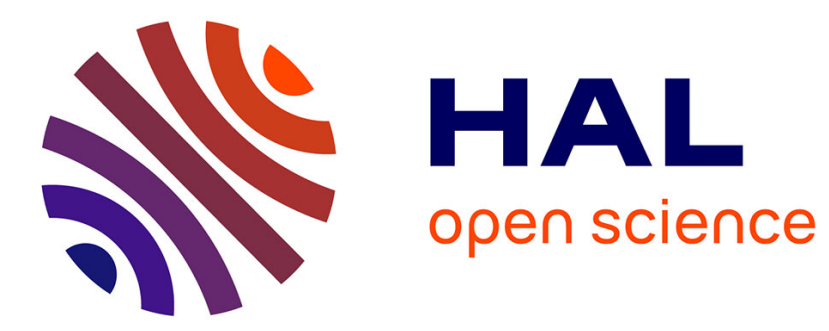

\title{
On the largest prime factors of consecutive integers in short intervals
}

\author{
Zhiwei Wang
}

\section{To cite this version:}

Zhiwei Wang. On the largest prime factors of consecutive integers in short intervals. Proceedings of the American Mathematical Society, 2017, 145 (8), pp.3211-3220. 10.1090/proc/13459 . hal-03283538

\section{HAL Id: hal-03283538 \\ https://hal.science/hal-03283538}

Submitted on 11 Jul 2021

HAL is a multi-disciplinary open access archive for the deposit and dissemination of scientific research documents, whether they are published or not. The documents may come from teaching and research institutions in France or abroad, or from public or private research centers.
L'archive ouverte pluridisciplinaire HAL, est destinée au dépôt et à la diffusion de documents scientifiques de niveau recherche, publiés ou non, émanant des établissements d'enseignement et de recherche français ou étrangers, des laboratoires publics ou privés. 


\title{
On the largest prime factors of consecutive integers in short intervals
}

\author{
Zhiwei Wang*
}

\section{Introduction}

For each integer $n \geqslant 1$, let $P(n)$ denote the largest prime factor of $n$ with the convention that $P(1)=1$. In 1930, Dickman [2] obtained the well-known result: the following asymptotic formula

$$
\Psi(x, y)=|\{n \leqslant x: P(n) \leqslant y\}| \sim x \varrho(u) \quad(u \geqslant 1)
$$

holds for $x \rightarrow \infty$ with $u=\log x / \log y$ fixed, where $\varrho(u)$ is the Dickman function. Furthermore, one conjectured that the largest prime factors of consecutive integers $n$ and $n+1$ are "independent events", that is to say, the density of integers $n$ with $P(n)<P(n+1)$ is 1/2. In this direction, in 1978 Erdős and Pomerance [3] proved that there exists a positive proportion of integers $n$ with $P(n)<P(n+1)$. More precisely, they proved that

Theorem (A). For $x \rightarrow \infty$, we have

$$
|\{n \leqslant x: P(n)<P(n+1)\}|>0.0099 x .
$$

Another important problem on consecutive integers is the following. Let $\left\{\varepsilon_{n}\right\}_{1 \leqslant n<N}$ be a finite sequence with each $\varepsilon_{n} \in\{0, \pm 1\}$, and write

$$
\frac{a}{b}=\prod_{1 \leqslant n<N}\left(\frac{n}{n+1}\right)^{\varepsilon_{n}}
$$

where the fraction is in its smallest terms, then define $A(N)$ as the maximal value of $a$ as $\left\{\varepsilon_{n}\right\}_{1 \leqslant n<N}$ runs through all possible $3^{N-1}$ sequences of $0, \pm 1$. In 1988 , Nicolas [7] showed that

$$
\log A(N) \leqslant\left\{\frac{2}{3}+o(1)\right\} N \log N
$$

and further a brief argument of M.Langevin is presented that

$$
\log A(N) \geqslant\{\log 4+o(1)\} N .
$$

In 2005, La Bretèche, Pomerance and Tenenbaum [1] improved the lower bound of $\log A(N)$ to the same order of magnitude as the upper bound:

*The author is supported by the China Scholarship Council. 
Theorem (B). For large $N$, we have

$$
\log A(N) \geqslant\{K+o(1)\} N \log N,
$$

where $K \approx 0.107005$ is a constant.

Further, they proved that for any fixed $c_{0} \in\left[0, \frac{1}{5}\right)$ and uniformly for $c \in\left[0, c_{0}\right], x \rightarrow \infty$,

$$
S(x, c) \leqslant 2 x \int_{0}^{c} \log \left(\frac{1-v}{1-v-2 c}\right) \frac{\mathrm{d} v}{1-v}+o(x),
$$

where $S(x, c)$ denotes the number of those integers $n$ not exceeding $x$ such that $P(n)>$ $x^{1-c}$ and $P(n+1)>x^{1-c}$. In fact, (1.2) is an easy consequence of (1.3). Besides, from (1.3) they also improved the result of Theorem (A) and obtained

Theorem (C). For $x \rightarrow \infty$, we have

$$
|\{n \leqslant x: P(n)<P(n+1)\}|>0.05544 x .
$$

In addition, in their paper they also indicated that the constant 0.05544 can be improved to 0.05866 by using more sophisticated sieve methods thanks to an observation of Fouvry. Indeed, when using sieve methods, La Bretèche, Pomerance and Tenenbaum disposed the sum of error term by the Bombieri-Vinogradov theorem, while Fouvry used a type of convolution of the Bombieri-Vinogradov theorem, which can improve the distribution level leading an improvement of the constant.

In this paper, we consider the largest prime factors of consecutive integers in short intervals $(x, x+y]$ with

$$
y=x^{\theta} \quad \text { with } \quad \frac{3}{5}<\theta \leqslant 1 .
$$

Throughout this paper, we denote by $\varepsilon$ an arbitrarily small positive constant, $\gamma$ Euler's constant and $p, p^{\prime}$ primes. For convenience, we write $\mathscr{L}=\log x$.

Our results are as follows.

Theorem 1. (i) Let

$$
\frac{3}{5}<\theta \leqslant 1 \quad \text { and } \quad 0<c<\min \left\{\frac{5 \theta-3}{2}, \theta-\frac{1}{2}\right\} .
$$

Then for $x \rightarrow \infty$ and $y=x^{\theta}$, we have

$$
|\{x<n \leqslant x+y: P(n)<P(n+1)\}| \geqslant\left\{g(\theta ; c)+o_{\theta, c}(1)\right\} y,
$$

where

$$
g(\theta ; c):=\log \frac{1}{1-c}-2 \int_{0}^{c} \frac{\log (1-v)^{-1}}{\theta-\frac{1}{2}-v} \mathrm{~d} v .
$$

(ii) For $\frac{3}{5}<\theta \leqslant 1$, there is a unique $c(\theta) \in\left(0, \min \left\{\frac{5 \theta-3}{2}, \theta-\frac{1}{2}\right\}\right)$ such that

$$
g(\theta):=\max _{0<c<\min \left\{\frac{5 \theta-3}{2}, \theta-\frac{1}{2}\right\}} g(\theta ; c)=g(\theta ; c(\theta))>0 .
$$

In particular, we have $g(1)>g(1 ; 0.1778)>0.1063$.

(iii) For $x \rightarrow \infty$, we have

$$
|\{n \leqslant x: P(n)<P(n+1)\}|>0.1063 x .
$$


Theorem 1.(iii) improves Fouvry's constant "0.05866" mentioned above.

We shall follow Fouvry's argument [1]. The starting point is the following inequality

$$
\sum_{\substack{x<n \leqslant x+y \\ P(n-1)<P(n)}} 1 \geqslant \sum_{\substack{x<n \leqslant x+y \\ P(n)>n^{1-c}}} 1-\sum_{\substack{x<n \leqslant x+y \\ P(n-1)>P(n)>n^{1-c}}} 1=: \mathcal{S}_{A}-\mathcal{S}_{B}
$$

with $0<c \leqslant \frac{1}{2}$. So we only need to give a lower bound of $\mathcal{S}_{A}$ and an upper bound of $\mathcal{S}_{B}$.

To estimate $\mathcal{S}_{A}$, we shall use the asymptotic formula about the distribution of friable numbers in short intervals.

For $\mathcal{S}_{B}$, if $n$ is counted by $\mathcal{S}_{B}$, then $n$ is of the form $n=a p+1=b p^{\prime}$ with $p>p^{\prime}>n^{1-c}$, namely $a<b \leqslant n^{c}$. So we have

$$
\begin{aligned}
\mathcal{S}_{B} & \leqslant\left|\left\{x<n \leqslant x+y: n=a p+1=b p^{\prime}, a<b \leqslant(x+y)^{c}\right\}\right| \\
& =\sum_{b \leqslant(x+y)^{c}}\left|\left\{x<n \leqslant x+y: n=a p+1=b p^{\prime}, a<b\right\}\right| \\
& =\sum_{b \leqslant(x+y)^{c}} \mid\{n \in \mathscr{A}(b): n \text { is prime }\} \mid,
\end{aligned}
$$

where

$$
\mathscr{A}(b):=\left\{\frac{a p+1}{b}: x<a p \leqslant x+y, a<b, a p+1 \equiv 0(\bmod b)\right\} .
$$

It is because of the substitution of " $a<x^{c}$ " with " $a<b$ " in the above sequence $\mathscr{A}(b)$ that we can improve Fouvry's constant "0.05866" to "0.1063". Then we will use RosserIwaniec's sieve $[5,6]$ to sieve $\mathscr{A}(b)$ by the following set of primes

$$
\mathscr{P}=\{p: p \text { is prime }\},
$$

so that we can give an upper bound of $\mathcal{S}_{B}$. In addition, we also need some generalized Bombieri-Vinogradov theorems in short intervals [9].

Theorem 2. For large $N$, we have

$$
\log A(N) \geqslant\{K+o(1)\} N \log N,
$$

where $K \approx 0.1108$ is a constant.

Theorem 2 improves the constant $K \approx 0.107005$ of Theorem (B).

Acknowledgement. This paper is directed by the author's advisors of thesis C. Dartyge and J. Wu. The author expresses his sincere gratitude to them for their lot of uesful and crucial advice. Also he thanks Professeur Wenguang Zhai for a crucial suggestion. 


\section{Lemmas}

Let $\mathcal{A}$ be a finite sequence of integers, $\mathcal{P}$ a set of primes, $z \geqslant 2$ a real number and $d$ a squarefree integer with all its prime factors belonging to $\mathcal{P}$, and denote

$$
\mathcal{A}_{d}:=\{a \in \mathcal{A}: d \mid a\}, \quad P(z):=\prod_{p<z, p \in \mathcal{P}} p
$$

We shall evaluate

$$
S(\mathcal{A} ; \mathcal{P}, z):=|\{a \in \mathcal{A}:(a, P(z))=1\}| .
$$

Assume that $\left|\mathcal{A}_{d}\right|$ may be written in the form

$$
\left|\mathcal{A}_{d}\right|=\frac{\omega(d)}{d} X+r(\mathcal{A}, d) \text { for } d \mid P(z)
$$

where $X$ is an approximation to $|\mathcal{A}|$ independent of $d, \omega$ is a multiplicative function satisfying $0<\omega(p)<p$ for $p \in \mathcal{P}, \omega(d) d^{-1} X$ is considered as a main term and $r(\mathcal{A}, d)$ is an error term which we expect is small on average over $d$.

The first lemma is a simple consequence of [5, Theorem 1].

Lemma 2.1. Suppose that there exists a constant $K \geqslant 2$ such that

$$
\prod_{w \leqslant p<v}\left(1-\frac{\omega(p)}{p}\right)^{-1}<\frac{\log v}{\log w}\left(1+\frac{K}{\log w}\right)
$$

for all $v>w \geqslant 2$. Then for any $D \geqslant z \geqslant 2$, we have

$$
S(\mathcal{A} ; \mathcal{P}, z) \leqslant X V(z)\left\{F(s)+O\left(\frac{1}{(\log y)^{1 / 3}}\right)\right\}+\sum_{d<D, d \mid P(z)}|r(\mathcal{A}, d)|,
$$

where $s:=\log D / \log z$ and

$$
V(z):=\prod_{p<z, p \in \mathcal{P}}\left(1-\omega(p) p^{-1}\right), \quad F(s):=2 \mathrm{e}^{\gamma} s^{-1} \quad(0<s \leqslant 3) .
$$

For $(a, q)=1$ and $\ell \geqslant 1$, we define

$$
\pi(z ; q, a, \ell)=\sum_{\substack{\ell p \leqslant z \\ \ell p \equiv a(\bmod q)}} 1
$$

The second lemma is [9, Theorem].

Lemma 2.2. Let $g(\ell)$ be an arithmetic function satisfying

$$
\sum_{\ell \leqslant x}|g(\ell)|^{2} \ell^{-1} \ll \mathscr{L}^{\lambda}
$$


for some positive constant $\lambda>0$. Define

$$
H(z, h ; q, a, \ell):=\pi(z+h ; q, a, \ell)-\pi(z ; q, a, \ell)-\frac{1}{\varphi(q)} \int_{z / \ell}^{(z+h) / \ell} \frac{\mathrm{d} t}{\log t} .
$$

Then for any $A>0$ and $\varepsilon>0$, there exists a constant $B=B(A, \lambda)>0$ such that the estimate

$$
\sum_{q \leqslant Q} \max _{(a, q)=1} \max _{h \leqslant y} \max _{x / 2<z \leqslant x}\left|\sum_{\substack{\ell \leqslant L \\(\ell, q)=1}} g(\ell) H(z, h ; q, a, \ell)\right| \ll_{A, \lambda, \varepsilon} y \mathscr{L}^{-A}
$$

holds uniformly for $\frac{3}{5}+\varepsilon \leqslant \theta \leqslant 1, Q=x^{\theta-1 / 2} \mathscr{L}^{-B}$ and $L=x^{(5 \theta-3) / 2-\varepsilon}$.

In particuliar, we can easily see that Lemma 2.2 covers the result of Perelli, Pintz and Salerno [8], which states that if $\pi(z ; q, a):=\pi(z ; q, a, 1)$, then for any $A>0$ we have

$$
\sum_{q \leqslant Q} \max _{(a, q)=1} \max _{h \leqslant y} \max _{x / 2<z \leqslant x}\left|\pi(z+h ; q, a)-\pi(z ; q, a)-\frac{1}{\varphi(q)} \int_{z}^{z+h} \frac{\mathrm{dt}}{\log t}\right| \ll y \mathscr{L}^{-A},
$$

uniformly for $\frac{3}{5}<\theta \leqslant 1$ and $Q=x^{\theta-1 / 2} \mathscr{L}^{-B}$, where $B=B(A)$ is a positive constant.

The third lemma is [4, Theorem 3].

Lemma 2.3. As $\Psi(x, y)$ is defined by (1.1), then the following asymptotic formula

$$
\Psi(x+z, y)-\Psi(x, y) \sim \frac{z}{x} \Psi(x, y) \sim z \varrho(u)
$$

holds uniformly for $1 \leqslant x / z \leqslant y^{5 / 12}$ and $\exp \left\{(\log \mathscr{L})^{5 / 3+\varepsilon}\right\} \leqslant y \leqslant x$.

\section{Proof of Theorem 1 and 2}

For Theorem 1, recall that $\mathcal{S}_{A}, \mathcal{S}_{B}, \mathscr{A}(b)$ and $\mathscr{P}$ are defined in (1.8), (1.9) and (1.10) respectively. First, we shall give an upper bound of $\mathcal{S}_{B}$, and then estimate $\mathcal{S}_{A}$.

For $\mathcal{S}_{B}$, we first sieve $\mathscr{A}(b)$ by $\mathscr{P}$, and then sum over $b$. Let $\theta$ and $c$ be two positive real numbers satisfying (1.4) respectively, and $z=x^{\theta-1 / 2} /\left(b \mathscr{L}^{B_{0}}\right)$, where $B_{0}$ is an appropriate positive constant. So for $b \leqslant(x+y)^{c}$ and $d \mid P(z)$, we have

$$
\begin{aligned}
\left|\mathscr{A}_{d}(b)\right| & =\left|\left\{\frac{a p+1}{b}: x<a p \leqslant x+y, a<b, a p+1 \equiv 0(\bmod b d)\right\}\right| \\
& =\sum_{a<b} \sum_{\substack{x<a p \leqslant x+y \\
a p+1 \equiv 0(\bmod b d)}} 1 .
\end{aligned}
$$


With the notation (2.1) we can write as follows

$$
\begin{aligned}
\left|\mathscr{A}_{d}(b)\right| & =\sum_{a<b} \sum_{\substack{x<a p \leqslant x+y \\
a p+1 \equiv 0(\bmod b d)}} 1 \\
& =\sum_{\substack{a<b \\
(a, b d)=1}} \frac{1}{\varphi(b d)} \int_{x / a}^{(x+y) / a} \frac{\mathrm{d} t}{\log t}+\sum_{\substack{a<b \\
(a, b d)=1}} H(x, y ; b d,-1, a) .
\end{aligned}
$$

For the first sum on the right hand side of (3.1), we have

$$
\begin{aligned}
\sum_{\substack{a<b \\
(a, b d)=1}} \frac{1}{\varphi(b d)} \int_{x / a}^{(x+y) / a} \frac{\mathrm{d} t}{\log t} & =\sum_{\substack{a<b \\
(a, b d)=1}} \frac{y}{a \varphi(b d)}\left\{\frac{1}{\log \frac{x}{a}}+O\left(\frac{1}{\log \frac{x}{a}}-\frac{1}{\log \frac{x+y}{a}}\right)\right\} \\
& =\left\{1+O\left(\frac{1}{\mathscr{L}}\right)\right\} \frac{y}{\varphi(b d)} \sum_{\substack{a<b \\
(a, b d)=1}} \frac{1}{a \log (x / a)}
\end{aligned}
$$

By the Möbius inversion, we have

$$
\begin{aligned}
\sum_{\substack{a<b \\
(a, b d)=1}} \frac{1}{\varphi(b d)} \int_{x / a}^{(x+y) / a} \frac{\mathrm{d} t}{\log t} & =\left\{1+O\left(\frac{1}{\mathscr{L}}\right)\right\} \frac{y}{\varphi(b d)} \sum_{a<b} \frac{1}{a \log (x / a)} \sum_{q \mid(a, b d)} \mu(q) \\
& =\left\{1+O\left(\frac{1}{\mathscr{L}}\right)\right\} \frac{y}{\varphi(b d)} \sum_{q \mid b d} \frac{\mu(q)}{q} \sum_{a q<b} \frac{1}{a \log (x / a q)} \\
& =\left\{1+O\left(\frac{1}{\mathscr{L}}\right)\right\} \frac{y}{\varphi(b d)}\left(S_{1}+S_{2}\right),
\end{aligned}
$$

where

$$
S_{1}:=\sum_{q \mid b d, q<\mathscr{L}^{9}} \frac{\mu(q)}{q} \sum_{a<b / q} \frac{1}{a \log (x / a q)}, \quad S_{2}:=\sum_{q \mid b d, q \geqslant \mathscr{L}^{9}} \frac{\mu(q)}{q} \sum_{a<b / q} \frac{1}{a \log (x / a q)} .
$$

Denoting by $\tau(n)$ the number of divisors of $n$, we have

$$
S_{2} \ll \sum_{q \mid b d, q \geqslant \mathscr{L}^{9}} q^{-1} \ll \tau(b d) \mathscr{L}^{-9} .
$$

And for $S_{1}$, by the partial summation we have

$$
\begin{aligned}
S_{1} & =\sum_{q \mid b d, q<\mathscr{L}^{9}} \frac{\mu(q)}{q}\left\{\int_{1}^{b / q} \frac{\mathrm{d} t}{t \log (x / t q)}+O\left(\frac{1}{\mathscr{L}}\right)\right\} \\
& =\sum_{q \mid b d, q<\mathscr{L}^{9}} \frac{\mu(q)}{q}\left\{\log \left(\frac{\log x-\log q}{\log x-\log b}\right)+O\left(\frac{1}{\mathscr{L}}\right)\right\} \\
& =\sum_{q \mid b d, q<\mathscr{L}^{9}} \frac{\mu(q)}{q}\left\{\log \left(\frac{\log x}{\log (x / b)}\right)+O\left(\frac{\log \mathscr{L}}{\mathscr{L}}\right)\right\},
\end{aligned}
$$


owing to the condition $q<\mathscr{L}^{9}$, we can separate the above $\log q$ from the main term. Thus we have

$$
\begin{aligned}
S_{1} & =\left(\sum_{q \mid b d} \frac{\mu(q)}{q}-\sum_{\substack{q \mid b d \\
q \geqslant \mathscr{L}^{9}}} \frac{\mu(q)}{q}\right)\left\{\log \left(\frac{\log x}{\log (x / b)}\right)+O\left(\frac{\log \mathscr{L}}{\mathscr{L}}\right)\right\} \\
& =\frac{\varphi(b d)}{b d}\left\{\log \left(\frac{\log x}{\log (x / b)}\right)+O\left(\frac{\log \mathscr{L}}{\mathscr{L}}\right)\right\}+O\left(\frac{\tau(b d)}{\mathscr{L}^{9}}\right) .
\end{aligned}
$$

So we infer from (3.1), (3.2), (3.3) and (3.4) that

$$
\left|\mathscr{A}_{d}(b)\right|=\frac{\omega(d)}{d} X+r(\mathscr{A}(b), d)
$$

with $\omega(d)=1$ and

$$
\begin{gathered}
X=\frac{y}{b} \log \left(\frac{\log x}{\log (x / b)}\right)\left\{1+O\left(\frac{\log \mathscr{L}}{\mathscr{L}}\right)\right\}, \\
r(\mathscr{A}(b), d)=\sum_{\substack{a<b \\
(a, b d)=1}} H(x, y ; b d,-1, a)+O\left(\frac{y \tau(b d)}{\varphi(b d) \mathscr{L}^{9}}\right) .
\end{gathered}
$$

Thus we can apply Lemma 2.1 with $D=z=x^{\theta-1 / 2} /\left(b \mathscr{L}^{B_{0}}\right)$ to write

$$
S(\mathscr{A}(b) ; \mathscr{P}, z) \leqslant\{1+o(1)\} \frac{2 X}{\log \left(x^{\theta-1 / 2} / b\right)}+\sum_{d<z, d \mid P(z)}|r(\mathscr{A}(b), d)|,
$$

where we have used Mertens' formula to evaluate

$$
V(z)=\prod_{p<x^{\theta-1 / 2} b^{-1} \mathscr{L}^{-B_{0}}}\left(1-\frac{1}{p}\right)=\frac{\mathrm{e}^{-\gamma}}{\log \left(x^{\theta-1 / 2} / b\right)}\left\{1+O\left(\frac{\log \mathscr{L}}{\mathscr{L}}\right)\right\} .
$$

From this and the trivial inequality

$$
\mid\{n \in \mathscr{A}(b): n \text { is prime }\} \mid \leqslant S(\mathscr{A}(b) ; \mathscr{P}, z)+z,
$$

we deduce

$$
\mathcal{S}_{B} \leqslant \sum_{b \leqslant(x+y)^{c}}(S(\mathscr{A}(b) ; \mathscr{P}, z)+z) \leqslant\{1+o(1)\} S_{3}+S_{4}+O\left(y \mathscr{L}^{-1}\right),
$$

where

$$
\begin{aligned}
S_{3} & :=\sum_{b \leqslant(x+y)^{c}} \frac{2 y}{b \log \left(x^{\theta-1 / 2} / b\right)} \log \left(\frac{\log x}{\log (x / b)}\right), \\
S_{4} & :=\sum_{b \leqslant(x+y)^{c}} \sum_{d<z}\left|\sum_{\substack{a<b \\
(a, b d)=1}} H(x, y ; b d,-1, a)\right|,
\end{aligned}
$$


and we have used the standard estimation $\sum_{m \leqslant x} \tau(m)^{2} / \varphi(m) \ll \mathscr{L}^{4}$ to bound

$$
y \mathscr{L}^{-9} \sum_{b \leqslant(x+y)^{c}} \sum_{d<x^{\theta-1 / 2} /\left(b \mathscr{L}^{\left.B_{0}\right)}\right.} \frac{\tau(b d)}{\varphi(b d)} \ll y \mathscr{L}^{-9} \sum_{m<x^{1 / 2} \mathscr{L}^{-B_{0}}} \frac{\tau(m)^{2}}{\varphi(m)} \ll y \mathscr{L}^{-5} .
$$

First we evaluate the error term $S_{4}$. By the Cauchy-Schwarz inequality, it follows that

$$
S_{4} \leqslant \sum_{m<x^{\theta-1 / 2} \mathscr{L}^{-B_{0}}} \tau(m)\left|\sum_{\substack{a<b \\(a, m)=1}} H(x, y ; m,-1, a)\right| \leqslant\left(S_{41} S_{42}\right)^{1 / 2},
$$

where

$$
\begin{aligned}
S_{41} & :=\sum_{m<x^{\theta-1 / 2} \mathscr{L}^{-B_{0}}}\left|\sum_{\substack{a<b \\
(a, m)=1}} H(x, y ; m,-1, a)\right|, \\
S_{42} & :=\sum_{m<x^{\theta-1 / 2} \mathscr{L}^{-B_{0}}} \tau(m)^{2}\left|\sum_{\substack{a<b \\
(a, m)=1}} H(x, y ; m,-1, a)\right| .
\end{aligned}
$$

For $S_{42}$, we use a trivial estimate and for $S_{41}$, we shall apply Lemma 2.2 with

$$
g(\ell)= \begin{cases}1 & \text { if } 0<\ell \leqslant b \\ 0 & \text { if } b<\ell \leqslant x^{(5 \theta-3) / 2-\varepsilon}\end{cases}
$$

since $b \leqslant(x+y)^{c} \leqslant x^{(5 \theta-3) / 2-\varepsilon}$ thanks to hypothesis (1.4). So we obtain

$$
\begin{aligned}
S_{4} & \ll\left(\frac{y}{\mathscr{L}^{A}}\right)^{1 / 2}\left(\sum_{m<x^{\theta-1 / 2} \mathscr{L}^{-B_{0}}} \tau(m)^{2} \sum_{a<b}\left\{\frac{y}{a m}+O(1)\right\}\right)^{1 / 2} \\
& \ll\left(\frac{y}{\mathscr{L}^{A}}\right)^{1 / 2}\left(y \mathscr{L}^{5}+b x^{\theta-1 / 2} \mathscr{L}^{-B_{0}+3}\right)^{1 / 2} \\
& \ll \frac{y}{\mathscr{L}^{A / 2-3}}
\end{aligned}
$$

since $y^{1 / 2} x^{(c+\theta-1 / 2) / 2} \ll y x^{-(1 / 2-c) / 2} \ll y$ with $c<\theta-\frac{1}{2} \leqslant \frac{1}{2}$.

Next we evaluate $S_{3}$. By partial summation we get

$$
\begin{aligned}
S_{3} & =2 y\{1+o(1)\} \int_{1}^{(x+y)^{c}} \log \left(\frac{\log x}{\log (x / t)}\right) \frac{\mathrm{d} t}{t \log \left(x^{\theta-1 / 2} / t\right)} \\
& =y\left\{2 \int_{0}^{c} \frac{\log (1-v)^{-1}}{\theta-\frac{1}{2}-v} \mathrm{~d} v+o_{\theta, c}(1)\right\} .
\end{aligned}
$$

So from (3.5), (3.6) and (3.7) we obtain

$$
\mathcal{S}_{B} \leqslant y\left\{2 \int_{0}^{c} \frac{\log (1-v)^{-1}}{\theta-\frac{1}{2}-v} \mathrm{~d} v+o_{c, \theta}(1)\right\} .
$$


Finally we evaluate $\mathcal{S}_{A}$. Noticing that $\frac{3}{5}<\theta \leqslant 1,0<c<\theta-\frac{1}{2} \leqslant \frac{1}{2}$ and $\varrho(u)=1-\log u$ for $1 \leqslant u \leqslant 2$, Lemme 2.3 gives us immediately

$$
\mathcal{S}_{A}=y\left\{\log \frac{1}{1-c}+o_{c, \theta}(1)\right\} .
$$

Now, the required inequality (1.5) follows from from (1.8), (3.8) and (3.9).

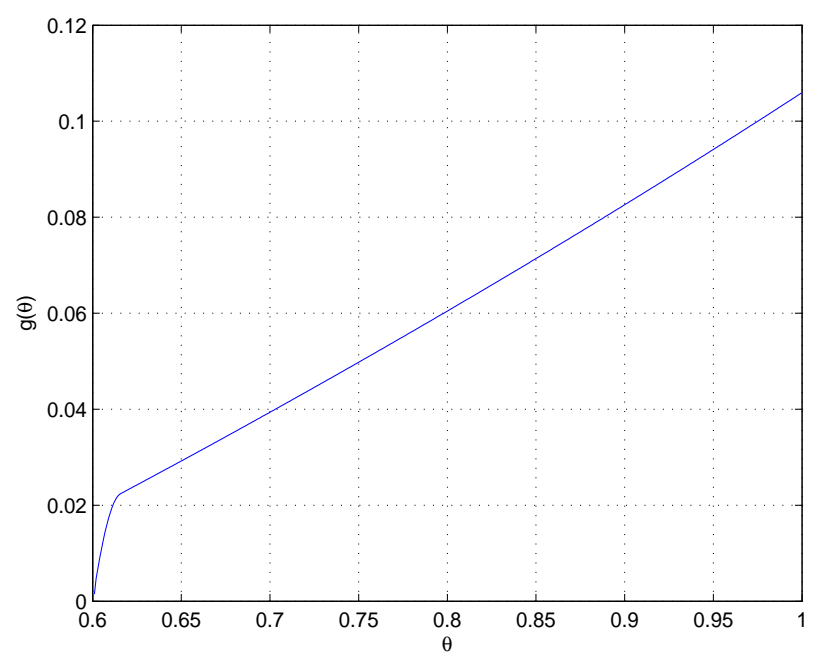

Figure 1: $g(\theta)$

We have

$$
\begin{aligned}
\frac{\partial g}{\partial c}(\theta ; c) & =\frac{1}{1-c}-2 \frac{\log (1-c)^{-1}}{\theta-\frac{1}{2}-c} \\
\frac{\partial^{2} g}{\partial c^{2}}(\theta ; c) & =-\frac{1}{1-c}\left(\frac{2}{\theta-\frac{1}{2}-c}-\frac{1}{1-c}\right)-2 \frac{\log (1-c)^{-1}}{\left(\theta-\frac{1}{2}-c\right)^{2}} .
\end{aligned}
$$

It's obvious that $\frac{\partial^{2} g}{\partial c^{2}}(\theta ; c)<0$ for $\frac{3}{5}<\theta \leqslant 1$ and $0 \leqslant c<\theta-\frac{1}{2}$. Thus $c \mapsto \frac{\partial g}{\partial c}(\theta ; c)$ is

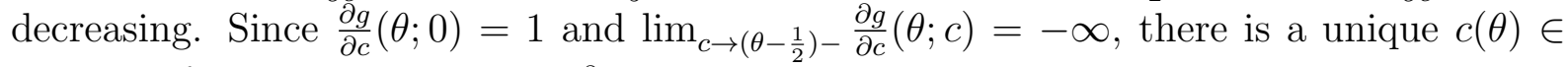
$\left(0, \min \left\{\frac{5 \theta-3}{2}, \theta-\frac{1}{2}\right\}\right)$ such that $\frac{\partial g}{\partial c}(\theta ; c(\theta))=0$ and

$$
g(\theta):=\max _{0 \leqslant c<\min \left\{\frac{50-3}{2}, \theta-\frac{1}{2}\right\}} g(\theta ; c)=g(\theta ; c(\theta))>g(\theta ; 0)=0
$$

for $\frac{3}{5}<\theta \leqslant 1$.

By Mathematica 9.0, we can find $c(1) \approx 0.1778$ and $g(1)=g(1, c(1))>0.1063$.

The assertion (iii) is an immediate consequence of the first two ones thanks to a simple dyadic summation.

This completes the proof of Theorem 1 . 
For Theorem 2, just as discussed in [1], the lower bound (1.11) is an easy consequence of (3.8) with $y=x$ and $0<c<1 / 2$. So we have

$$
\log A(N) \geqslant 2 N(\log N)\{g(c)+o(1)\}
$$

where

$$
g(c):=c-(1-c) f(c)-\int_{0}^{c} f(u) \mathrm{d} u, \quad \text { with } f(u):=4 \int_{0}^{u} \frac{\log (1-v)^{-1}}{\frac{1}{2}-v} \mathrm{~d} v .
$$

By Mathematica 9.0, we can find

$$
K:=\max _{0<c<1 / 2} 2 g(c) \approx 0.1108 .
$$

This completes the proof of Theorem 2 .

\section{References}

[1] R. de la Bretèche, C. Pomerance, and G. Tenenbaum. Products of ratios of consecutive integers. Ramanujan J., 9:131-138, 2005.

[2] K. Dickman. On the frequency of numbers containing prime factors of a certain relative magnitude. Ark. Mat. Astr. Fys., 22(A10):1-14, 1930.

[3] P. Erdös and C. Pomerance. On the largest prime factors of $n$ and $n+1$. Aequationes Math., 17(2-3):311-321, 1978.

[4] A. Hildebrand. On the number of positive integers $\leqslant x$ and free of prime factors $>y$. J. Number Theory, 22:289-307, 1986.

[5] H. Iwaniec. A new form of the error term in the linear sieve. Acta Arith., 37:307-320, 1980.

[6] H. Iwaniec. Rosser's sieve. Acta Arith., 36:171-202, 1980.

[7] J.-L. Nicolas. Nombres hautement composés. Acta Arith., 49:395-412, 1988.

[8] A. Perelli, J. Pintz, and S. Salerno. Bombieri's theorem in short intervals. Ann. Scvola Normale Sup. Pisa, 11(4):529-539, 1985.

[9] J. Wu. Théorèmes généralisés de Bombieri-Vinogradov dans les petits intervalles. Quart. J. Math. Oxford, 44(2):109-128, 1993.

Institut Élie Cartan, Université de Lorraine, B.P. 70239, 54506 Vandoeuvre-lès-Nancy Cedex, France

E-mail address: zhiwei.wang@univ-lorraine.fr 